\title{
A new method for the study of essential fatty acid requirements in fish larvae
}

\author{
Sofia Morais* and Luís E. C. Conceição \\ CCMAR, Universidade do Algarve, Campus de Gambelas, 8005-139 Faro, Portugal \\ (Received 11 July 2008 - Revised 3 September 2008 - Accepted 3 September 2008 - First published online 30 October 2008)
}

This study describes a methodology with potential application in the estimation of essential fatty acid (EFA) requirements of fish larvae. Senegalese sole (Solea senegalensis) larvae were fed, from 16 days after hatching (DAH), on Artemia enriched with different oils, inducing graded dietary concentrations of DHA: (1) soyabean oil, containing no measurable amounts of DHA (NDHA); (2) fish oil, inducing a medium DHA level (MDHA, $3 \mathrm{~g}$ DHA/100 g fatty acids); and (3) a mixture of Easy DHA Selco and Microfeed, resulting in high DHA content (HDHA, $8 \mathrm{~g} / 100 \mathrm{~g}$ ). At $28 \mathrm{DAH}$ a metabolic trial was conducted where larvae were tube fed $\left[1-{ }^{14} \mathrm{C}\right] \mathrm{DHA}$, in order to determine its absorption, retention in the gut and body tissues, as well as its oxidation. At 23 DAH the HDHA treatment induced a significantly higher larval growth, while at 32 DAH significant differences were only found between the NDHA and HDHA treatments. The absorption of tube-fed [1- ${ }^{14}$ C]DHA was extremely high (94-95\%) and independent of feeding regime. However, in larvae fed NDHA Artemia, a significantly higher amount of label was retained in the gut compartment and a concurrently lower retention was measured in the body. A significantly higher proportion of the absorbed DHA label was oxidized in larvae fed HDHA, compared to NDHA. Based on these results, we suggest that increasing dietary supply of DHA above the larval requirement level results in its increased oxidation for energy purposes and we propose potential applications of the tube feeding methodology using radiolabelled EFA in conjunction with dose-response studies.

Essential fatty acids: Fish larvae nutrition: Requirements: Metabolism

Marine fish resemble all other studied vertebrates in the fact that they need a dietary supply of PUFA, particularly DHA (22:6n-3), EPA $(20: 5 n-3)$ and arachidonic acid (20:4n-6) for their normal growth and development ${ }^{(1)}$. These are essential fatty acids (EFA) for marine fish, which cannot biosynthesize them de novo nor from shorter-chain precursors such as $18: 3 n-3$ or $18: 2 n-6^{(1,2)}$. Their biochemical, cellular and physiological functions are the same in fish as in other vertebrates, where they have a central role in maintaining the structural and functional integrity of cell membranes and as precursors of important metabolites, such as eicosanoids ${ }^{(1)}$. DHA, in particular, has high biological value as one of the major PUFA constituents of cell membranes and is the most abundant fatty acid (FA) in the neural and visual tissue of fish $^{(1,3,4)}$. Fish tissues, in general, are even richer in DHA than mammalian tissues and fish brain lipids are characterized by a higher degree of unsaturation and increased percentages of DHA and EPA, compared to the rat ${ }^{(3,4)}$. Dietary deficiencies of DHA leading to a depletion of DHA in the phospholipids of the rod cells of the retina have been associated with a decreased ability to capture preys at low light intensity in juvenile herring, denoting an impaired vision and/or neural function ${ }^{(4)}$. On the other hand, neural or visual malfunctioning may be one of the factors responsible for the abnormal malpigmentation which is common in cultured flatfish larvae ${ }^{(2,5)}$.
Membranes of the melanophores determining pigmentation may also be directly affected by DHA deprivation ${ }^{(2)}$. Finally, dietary deficiencies of DHA have been related to poor growth, survival, abnormal behaviour and higher susceptibility to stress and disease (i.e. reduced immunity) of several fish larvae species ${ }^{(6-8)}$.

The need to provide adequate dietary levels of DHA for early developing fish with unusually high growth rates $\left(10-100 \%\right.$ per d) and large developmental demands ${ }^{(9)}$ is therefore unquestionably one of the highest priorities in larval nutrition, which is reflected in the large number of studies focusing on the determination of DHA requirements of different fish species ${ }^{(1,2)}$. Dose-response approaches are commonly used in mammalian and juvenile and adult fish nutrition studies but have been very difficult to adapt to marine fish larvae, given that they are initially fed on live prey with a variable and extremely difficult to control nutritional composition. However, the development of new products and live food enrichment technologies ${ }^{(10)}$ has recently enabled the use of dose-response designs to study the EFA requirements of marine fish larvae ${ }^{(8,11,12)}$. Studies examining the biological response of fish larvae to key indicators of larval performance such as survival, growth, pigmentation and stress resistance, to feeding live prey (mostly rotifers and Artemia) enriched with graded concentrations of the

Abbreviations: DAH, days after hatching; EFA, essential fatty acids; FA, fatty acids; HDHA, high DHA; IAA, indispensable amino acids; MDHA, medium DHA; NDHA, no dietary DHA.

* Corresponding author: Dr Sofia Morais, Institute of Aquaculture, University of Stirling, Stirling FK9 4LA, UK, fax +44 1786 472133, email sofia.morais@ stir.ac.uk 
EFA of interest or with a gradient of EFA ratios (e.g. DHA:EPA or EPA:arachidonic acid) will likely become more common. However, these parameters might not be the most sensitive to determine an exact EFA requirement.

In mammals, in order to determine an indispensable amino acid (IAA) requirement, the indicator amino acid concept is commonly employed, resulting in very accurate estimates ${ }^{(13)}$. This method is based on the idea that when one IAA is deficient for protein synthesis, then all other IAA (including a labelled indicator) will be oxidized. However, when increasing amounts of the limiting IAA are included in the diet, the oxidation of the indicator IAA will decrease, until no further change is observed in the indicator oxidation, signifying that the requirement for the limiting IAA is met ${ }^{(13)}$. EFA, on the other hand, and DHA in particular, given their prominent structural role, are mostly retained in body tissues and are a poor substrate for the energy-generating $\beta$-oxidation system $^{(2)}$. Therefore, in the present study it was hypothesized that an increase in the dietary supply of DHA above larval requirements would result in its increased catabolism or oxidation, enabling in this way an accurate and quick estimation of DHA dietary requirements. In order to test this assumption, Senegalese sole (Solea senegalensis Kaup, 1858) larvae were fed Artemia enriched with different oil emulsions resulting in three different DHA concentrations. The effects on growth were analysed and a complementary metabolic trial was additionally performed, enabling the determination of not only the total absorption and tissue retention of ${ }^{14} \mathrm{C}$-labelled DHA that was tube fed but also its metabolic fate (i.e. how much was oxidized for the production of energy).

\section{Materials and methods}

\section{Larval rearing and experimental diets}

Larvae were obtained from IPIMAR Aquaculture Research Centre (Olhão, Portugal) at 14 days after hatching (DAH), with an average wet weight of 3.73 (SD 0.17) $\mathrm{mg}(n 5$, twenty pooled larvae). Until this age they had been fed Artemia enriched in a mixture of microalgae (Nannochloropsis sp. and Isochrysis sp.). At this time the larvae had already settled and were transferred to a recirculation system of nine 3-litre flat bottom trays, which were stocked with 200 larvae each. During two acclimation days all trays were fed Artemia enriched for $12 \mathrm{~h}$, at 250 nauplii/ml, with a mixture of Easy DHA Selco (INVE Aquaculture NV, Dendermonde, Belgium; two doses, at 0 and $9 \mathrm{~h}$, according to manufacturer's instructions) and Microfeed (EWOS, Bathgate, UK; $0.4 \mathrm{~g} / \mathrm{l}$ in the same two doses). From $16 \mathrm{DAH}$ onwards the larvae were fed one of three experimental treatments, in triplicate trays: (1) Artemia enriched on a soyabean oil emulsion, containing no dietary DHA (NDHA); (2) Artemia enriched on a fish oil emulsion, a medium DHA regime (MDHA); and (3) Artemia enriched on a mixture of Easy DHA Selco and Microfeed, containing a high DHA level (HDHA). The HDHA Artemia was enriched in the conditions described earlier, while the enrichments using soyabean oil and fish oil emulsions were conducted at a density of 150 nauplii/ $/ \mathrm{ml}$, during $16 \mathrm{~h}$, with $0.6 \mathrm{~g} / \mathrm{l}$ being added in two doses $(0.4 \mathrm{~g} / \mathrm{l}$ at $0 \mathrm{~h}$ and $0.2 \mathrm{~g} / \mathrm{l}$ at $8 \mathrm{~h})$. A single batch of enriched Artemia from each treatment was produced and was kept frozen for the duration of the experiment. Larvae were fed frozen enriched Artemia, after thawing in seawater, in excess to satiation, three times daily. Photoperiod was $12 \mathrm{~h}$ light $-12 \mathrm{~h}$ dark, salinity was kept at 33 and temperature varied between 20.5 and $22^{\circ} \mathrm{C}$. At 23 and $32 \mathrm{DAH}$, twenty and twenty-four larvae, respectively, were collected from each tray, flash frozen in liquid nitrogen and later freeze-dried in a Savant VLP120 ValuPump (Savant Instruments Inc., Holbrook, NY, USA), for the determination of individual dry weight.

\section{Fatty acid analysis}

Triplicate samples of Artemia from each treatment were collected into liquid nitrogen, for the determination of FA composition. In addition, at $32 \mathrm{DAH}$, a pool of twenty larvae was collected from each tray and stored in liquid nitrogen until FA analysis. Lipids were extracted with a chloroform-methanol $(2: 1, \mathrm{v}: \mathrm{v})$ mixture ${ }^{(14)}$. The FA methyl esters were obtained by transesterification with $1 \%$ sulphuric acid in methanol and were purified by adsorption chromatography on NH2 sep-pack cartridges (Waters, S.A., Milford, MA, USA $)^{(15)}$, and separated and quantified by GLC following the conditions described by Izquierdo et al. ${ }^{(16)}$. FA methyl esters were identified by comparison to external standards.

\section{Tube feeding experimental set-up and metabolic trial}

A tube feeding trial was conducted at $28 \mathrm{DAH}$, using the methodology and experimental set-up described by Rønnestad et al. ${ }^{(17)}$ and Morais et al. ${ }^{(18)}$, to examine the absorption and metabolism of a $\left[1-{ }^{14} \mathrm{C}\right] \mathrm{DHA}$ label (ARC American Radiolabelled Chemicals Inc., St Louis, MO, USA; 3.7 MBq/ml), after an incubation period of $24 \mathrm{~h}$. Briefly, in this set-up each larva is placed in a separate $20 \mathrm{ml}$ scintillation vial (Sigma-Aldrich, St Louis, MO, USA) containing $8 \mathrm{ml}$ seawater. The incubation vials are sealed well and connected to metabolic trap vials containing $5 \mathrm{ml} \mathrm{KOH}(0.5 \mathrm{M})$ that retain the ${ }^{14} \mathrm{CO}_{2}$ resulting from oxidation or catabolism, which is gradually released from the incubation water after it has been acidified with $1 \mathrm{ml} \mathrm{HCl}(0 \cdot 1 \mathrm{M})$.

A mixture containing the label was prepared by adding the $\left[1-{ }^{14} \mathrm{C}\right] \mathrm{DHA}$ to fish oil, to obtain a specific activity of $222 \mathrm{dpm} / \mathrm{nl}$ (or $3.7 \mathrm{~Bq} / \mathrm{nl}$ ), followed by evaporation of all traces of solvent by flushing with $\mathrm{N}_{2}$. The mixture was then stored at $-20^{\circ} \mathrm{C}$ until use. On the day before the trial, 30 min after the last meal of the day, six larvae were removed from each triplicate tray and pooled in smaller trays that were transported to the radioisotope experimental room, where the larvae were acclimated and kept unfed overnight. Temperature and salinity were maintained at $22^{\circ} \mathrm{C}$ and 35 , respectively, and the trial was conducted under continuous illumination. Before tube feeding approximately 15000 Artemia metanauplii of each treatment were added to the corresponding tray and larvae were allowed to feed for $30 \mathrm{~min}$. Twelve larvae from each treatment were first sedated in $29.4 \mu \mathrm{g} / \mathrm{ml}$ solution of MS-222 (Sigma-Aldrich) and then tube fed $23 \mathrm{nl}$ $\left[1-{ }^{14} \mathrm{C}\right] \mathrm{DHA}$ mixture. Each larva was removed from the incubation set-up $24 \mathrm{~h}$ after tube feeding, rinsed in clean saltwater, killed instantaneously by spinal cord severing with a scalpel and the gut was separated from the remaining body by dissection. Both compartments were transferred separately to $6 \mathrm{ml}$ 
scintillation vials (Sarstedt, Rio de Mouro, Portugal) and were solubilized in $200 \mu 130 \%$ (w/v) hydrogen peroxide (SigmaAldrich) at $60^{\circ} \mathrm{C}$ for $24 \mathrm{~h}$. After cooling, $4 \mathrm{ml}$ scintillation cocktail (Ultima Gold XR, Packard Bioscience, Monza, Italy) were added to each $6 \mathrm{ml}$ vial. The scintillation vials containing the incubation seawater (including the evacuated label) and the metabolic trap (including the ${ }^{14} \mathrm{CO}_{2}$ resulting from oxidation) were prepared for scintillation counting by adding 12 or $10 \mathrm{ml}$, respectively, of Ultima Gold $\mathrm{XR}^{(17,18)}$. The samples were counted on a Beckman LS 6000IC (Beckman Instruments Inc., Fullerton, CA, USA) liquid scintillation counter and the results are presented as a percentage of dpm in each compartment, in relation to the total counts.

\section{Statistical analysis}

The differences between larvae submitted to different dietary treatments, in terms of DHA content and growth, as well as the distribution of label in each compartment after tube feeding, were compared through one-way ANOVA, using the software Statistica 6 (StatSoft Inc., Tulsa, OK, USA). The assumption of homogeneity of variance was checked using the Bartlett's test and a significance level of 0.05 was employed $^{(19)}$. Percentage data from the tube feeding trial was $\arcsin \left(x^{1 / 2}\right)$ transformed. When significant differences were found, the Tukey HSD multiple range test was performed. All data are given as means and standard deviations.

\section{Results}

As expected, a gradient of DHA supply was accomplished by the different Artemia enrichments, with Artemia enriched on a soyabean oil emulsion (NDHA) having no measurable amounts of DHA, while the enrichments using fish oil (MDHA) or a mixture of Easy DHA Selco and Microfeed (HDHA) resulted in Artemia containing 3.0 and $8.1 \mathrm{~g} \mathrm{DHA} /$ $100 \mathrm{~g}$ FA, respectively (Table 1). At $32 \mathrm{DAH}$, the DHA content of the larvae reflected that of their diet, with significant differences $(P<0.001)$ being found between the larvae fed different diets (Table 1).

Larval growth, assessed by dry weight, was measured at 23 DAH, i.e. $7 \mathrm{~d}$ after start of feeding on the experimental diets,

Table 1. DHA content of the enriched Artemia and of 32 days after hatching $(\mathrm{DAH})$ larvae ( $n 3$, twenty pooled larvae), and larval dry weight $(\mathrm{mg})$ at 23 and $32 \mathrm{DAH}^{*}$

(Mean values and standard deviations)

\begin{tabular}{|c|c|c|c|c|c|c|}
\hline & \multicolumn{2}{|c|}{$\mathrm{NDHA}$} & \multicolumn{2}{|c|}{ MDHA } & \multicolumn{2}{|c|}{$\mathrm{HDHA}$} \\
\hline & Mean & SD & Mean & SD & Mean & SD \\
\hline \multicolumn{7}{|c|}{ DHA content (g DHA/100 g fatty acid) } \\
\hline Artemia & ND & & $3 \cdot 0$ & & $8 \cdot 1$ & \\
\hline Larvae & $1 \cdot 7^{\mathrm{a}}$ & 0.1 & $6 \cdot 7^{b}$ & 0.2 & $10 \cdot 4^{\mathrm{c}}$ & 0.5 \\
\hline \multicolumn{7}{|c|}{ Larval dry weight (mg) } \\
\hline $23 \mathrm{DAH}(n 60)$ & $1.53^{a}$ & 0.30 & $1.68^{\mathrm{a}}$ & 0.35 & $1.92^{b}$ & 0.36 \\
\hline $32 \mathrm{DAH}(n 72)$ & $4.64^{\mathrm{a}}$ & 1.05 & $5 \cdot 18^{a b}$ & 1.23 & $5 \cdot 30$ & 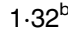 \\
\hline
\end{tabular}

HDHA, high DHA; MDHA, medium DHA; ND, not detected by GLC analysis; NDHA, no dietary DHA.

a,b,c Mean values within a row with unlike superscript letters were significantly different $(P<0.05)$.

${ }^{*}$ For details of procedures, see Materials and methods. when significant differences $(P<0 \cdot 001)$ were found between the HDHA and the other two treatments (Table 1). At 32 DAH, significant differences were found only between the NDHA and HDHA treatments $(P<0.05$; Table 1$)$.

The absorption and metabolic trial conducted at $28 \mathrm{DAH}$ revealed no significant differences in DHA absorption (total tube fed minus evacuated into the incubation water), $24 \mathrm{~h}$ after tube feeding. DHA was thus highly absorbed (94-95\% of total label tube fed), independently of the feeding regime (Fig. 1). In terms of label retention in the tissues, a significantly higher amount of label was retained in the gut compartment of larvae fed NDHA diet $(P<0.001)$, compared to the other two treatments, and a concurrently lower retention was measured in the body compartment of larvae fed this diet $(P<0.01)$, although it was only significantly different from the MDHA treatment (Fig. 1). As for the amount of ${ }^{14} \mathrm{CO}_{2}$ found in the metabolic trap, corresponding to the oxidized label, significant differences were found between the diets $(P=0.001)$, with a significantly lower amount of label being oxidized by larvae fed the NDHA diet than those fed HDHA, while an intermediate and non-significantly different amount of label was oxidized by MDHA-fed larvae (Fig. 1).

\section{Discussion}

The larval growth of Senegalese sole reflected the dietary level of DHA, although significant differences were only found between larvae fed Artemia containing no measurable amounts of DHA (NDHA) and the treatment containing higher levels of DHA (HDHA), after $16 \mathrm{~d}$ of feeding on the experimental treatments. It is believed that Senegalese sole larvae probably have lower DHA requirements during their larval stage than other marine fish species ${ }^{(11,20)}$. Villalta et al. ${ }^{(11)}$ hypothesize that the low DHA requirements of Senegalese sole may be explained by a predominance of EPA rather than DHA in the benthic fauna, which is the natural diet of this species after settlement.

In terms of larval DHA composition, it closely reflected the quantitative dietary supply, as is commonly found in fish larvae and has been described before for Senegalese sole larvae ${ }^{(20)}$. This indicates a high efficiency of absorption and retention of this EFA, as has been shown in the present study (94-95\% of total label absorbed, of which $89-94 \%$ was retained in the gut and body tissues) and has been demonstrated previously ${ }^{(18)}$. The present results are not surprising, having in mind the essential and structural role of $\mathrm{DHA}^{(1,2)}$, and strengthen the idea that marine fish larvae are extremely efficient in utilizing their dietary supply of EFA, possibly through a series of physiological mechanisms such as the high specificity of digestive enzymes towards $n$-3 PUFA ${ }^{(21)}$ and of the presumed higher affinity of cytosolic FA-binding proteins and higher rate of esterification of long-chain PUFA, as well as their preferential incorporation into polar lipids ${ }^{(22,23)}$.

DHA has been formerly considered an ideal quantitative marker of FA absorption, resulting from its minimal degradation, synthesis or conversion through elongation-desaturation activities ${ }^{(24)}$. This condition is also particularly useful in the type of trial conducted in the present experiment, as it can be reasonably assumed that the radiolabelled tracer found in each compartment corresponds to the FA which was tube fed. In terms of assimilation of the DHA label, the 


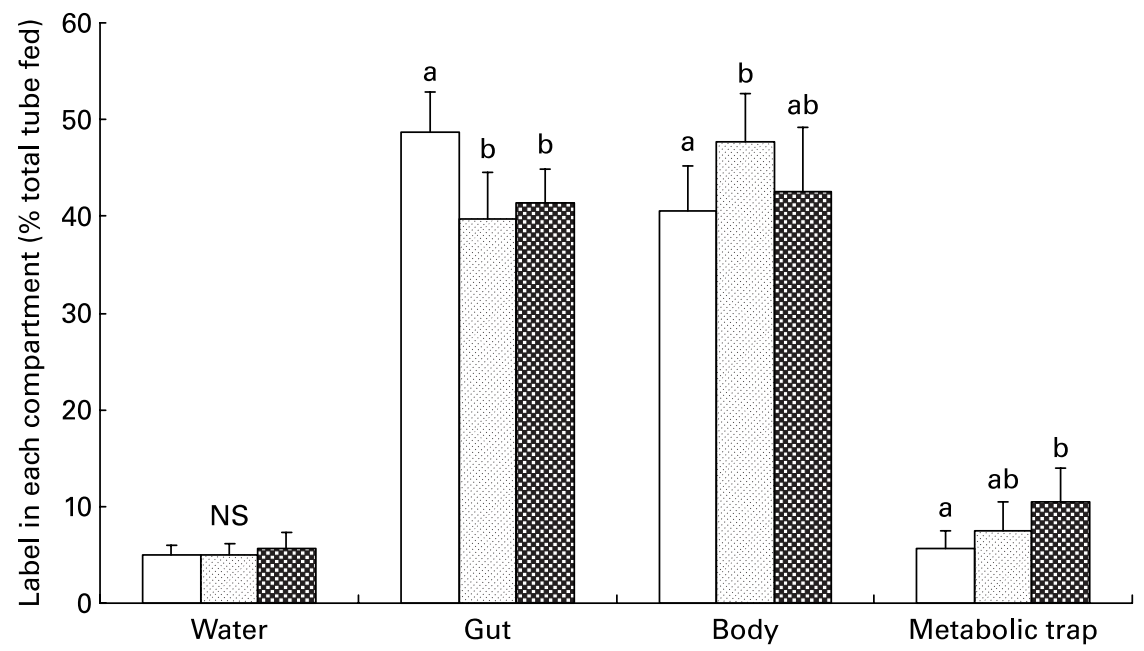

Fig. 1. Label in each compartment (\% total tube fed), $24 \mathrm{~h}$ after tube feeding $23 \mathrm{nl}$ of a fish oil mixture containing [1- $\left.{ }^{14} \mathrm{C}\right] \mathrm{DHA}$ to 28 days after hatching Senegalese sole larvae that were previously fed enriched Artemia containing graded concentrations of DHA (DHA/100 g fatty acid: $\square, 0 \mathrm{~g} ;{ }^{\circ}, 3 \mathrm{~g}$; 网, $8 \mathrm{~g}$ ). Values are means with their standard deviations depicted by vertical bars $(n 12) .{ }^{a, b}$ Mean values within each compartment with unlike letters were significantly different $(P<0.05)$.

results showed that when larvae were fed a diet void of DHA a significantly higher amount of DHA was incorporated into the gut and was concomitantly less in the remaining dissected body. This was certainly related to the state of malnutrition of these larvae but it remains unclear whether the results might be explained by a preferential incorporation of dietary DHA into the membranes of the gastrointestinal mucosa or, alternatively, by a kinetic effect of slowed down intracellular transport, under nutritional deficiency conditions.

As speculated, the assumption that an increase in the dietary supply would result in its increased oxidation for energy purposes proved to be true. In fact, a rise in the dietary DHA content led to significant differences in larval metabolism, with a significantly higher proportion of the absorbed DHA label being catabolized in larvae fed HDHA, compared to NDHA. For the MDHA treatment the catabolism was intermediate but not significantly different from NDHA or HDHA. The metabolic trial thus supports the growth results at $32 \mathrm{DAH}$, which appear to indicate that for Senegalese sole larvae fed enriched Artemia, after settlement, the requirements for DHA are probably not higher than $3 \mathrm{~g}$ DHA/100 g total FA. When larval growth was analysed at $23 \mathrm{DAH}$, only $7 \mathrm{~d}$ after the start of the dietary experiment, the results might have pointed to a higher DHA requirement (of around $8 \mathrm{~g} \mathrm{DHA}$ / $100 \mathrm{~g}$ total FA), since significant differences were only noted between HDHA-fed larvae and the remaining treatments. This may mean that DHA requirements of Senegalese sole larvae decrease with age and stage of development, or that the metabolic trial presented here might give an earlier indication of larval requirements, compared to other biological indicators, such as survival, growth, pigmentation and stress resistance. Nonetheless, further experiments are required using a larger number of dietary treatments providing graded levels of DHA, in order to narrow down the Senegalese sole larval requirements for this EFA. If a finer gradient of dietary DHA supply is tested the requirement may be estimated more accurately by the inflection point where the oxidation of the label significantly increases. This breakpoint might be identified using bi-phase linear regression analysis, as is done for
IAA requirement studies applying the indicator amino acid oxidation concept ${ }^{(13)}$.

In conclusion, the present study describes a methodology, based on tube feeding of a radioactive FA tracer and by following its metabolic fate, which may be used to estimate larval EFA requirements, in conjunction with dose-response studies. Growth has normally been used as an indicator to estimate requirements, as it normally reflects well dietary deficiencies. However, dose-response studies, which are becoming more common in larval nutritional research, may benefit from including a metabolic study of this type, particularly for narrowing down EFA requirements, as growth may not be a sufficiently sensitive parameter to discriminate between dietary treatments having close levels of EFA. In addition, it might be speculated that the metabolic response to a dietary supply of DHA in excess to requirements, such as an increased oxidative utilization for energy production, could be visible earlier than the physiological consequences of an unbalanced diet, although this remains to be shown. On the other hand, by tube feeding different EFA, such as DHA, EPA and arachidonic acid, to larvae fed a single set of diets differing in the relative levels and proportions between the different EFA it might be possible to estimate the requirement for multiple EFA simultaneously, in a single trial. Additionally, competitive interactions between dietary EPA and arachidonic acid and optimal ratios for eicosanoid production might also be addressed using this methodology, possibly even refining it further to include different body compartments of interest. Finally, one of the main interests of this technique may well be in studies of the effect of broodstock nutrition on early larval performance. For instance, it might be possible to correlate dietary levels of specific EFA in broodstock diets and the egg and early larvae FA composition with larval metabolism, before any effects on larval performance and quality become visible. However, most of these suggested potential applications of the methodology described here remain untested and should be explored in the next few years, hopefully helping to advance knowledge in the field of marine fish larvae nutrition. 


\section{Acknowledgements}

S. M. was supported by 'Fundação para a Ciência e a Tecnologia', Portugal (SFRH/BD/4902/2001). The experiments were supported by the Project PROMAR/SP5.P117/03 (programme INTERREG IIIA, co-funded by FEDER, European Commission), and comply with the current laws in Portugal, the country in which the present work was conducted. The authors are very grateful to Dr Marisol Izquierdo and Carmen Quintana, from Grupo de Investigación en Acuicultura, ULPGC and ICCM, Las Palmas de Gran Canaria, Spain, for performing the fatty acid analysis. The authors would also like to acknowledge the IPIMAR Aquaculture Research Centre (Olhão, Portugal) for the supply of Senegalese sole larvae. The authors declare no conflicts of interest. S. M. was responsible for designing and conducting the experiment, as well as writing the manuscript, while L. E. C. C. collaborated in the experimental design and manuscript preparation.

\section{References}

1. Sargent J, Bell G, McEvoy L, et al. (1999) Recent developments in the essential fatty acid nutrition of fish. Aquaculture $\mathbf{1 7 7}$ $191-199$

2. Sargent J, McEvoy L, Estevez A, et al. (1999) Lipid nutrition of marine fish during early development: current status and future directions. Aquaculture 179, 217-229.

3. Tocher DR \& Harvie DG (1988) Fatty acid compositions of the major phosphoglycerides from fish neural tissues; (n-3) and (n-6) polyunsaturated fatty acids in rainbow trout (Salmo gairdneri) and cod (Gadus morhua) brains and retinas. Fish Physiol Biochem 5, 229-239.

4. Bell MV, Batty RS, Dick JR, et al. (1995) Dietary deficiency of docosahexaenoic acid impairs vision at low light intensities in juvenile herring (Clupea harengus L.). Lipids 30, 443-449.

5. Estévez A \& Kanazawa A (1996) Fatty acid composition of neural tissues of normally pigmented and unpigmented juvenile of Japanese flounder using rotifer and Artemia enriched in n-3 HUFA. Fish Sci 62, 88-93.

6. Watanabe $T$ (1993) Importance of docosahexaenoic acid in marine larval fish. J World Aquac Soc 24, 152-161.

7. Furuita H, Takeuchi T \& Uematsu K (1998) Effects of eicosapentaenoic and docosahexaenoic acids on growth, survival and brain development of larval Japanese flounder (Paralichthys olivaceus). Aquaculture 161, 269-279.

8. Bransden MP, Cobcroft JM, Battaglene SC, et al. (2005) Dietary 22:6n-3 alters gut and liver structure and behaviour in larval striped trumpeter (Latris lineata). Aquaculture 248, 275-285.

9. Conceição LEC, Dersjant-Li Y \& Verreth JAJ (1998) Cost of growth in larval and juvenile African catfish (Clarias gariepinus) in relation to growth rate, food intake and oxygen consumption. Aquaculture 161, 95-106.

10. Kolkovski S (2005) Use of 'tailor-made' live-feed enrichments and additives in fish larvae nutritional research. In Larvi 2005, 4th Fish \& Shellfish Larviculture Symposium. European Aquaculture Society Special Publication no. 36, pp. 242-245 [CI Hendry, G Van Stappen, M Wille and P Sorgeloos, editors]. Oostende, Belgium: European Aquaculture Society.

11. Villalta M, Estévez A, Bransden MP, et al. (2005) The effect of graded concentrations of dietary DHA on growth, survival and tissue fatty acid profile of Senegal sole (Solea senegalensis) larvae during the Artemia feeding period. Aquaculture 249, 353-365.

12. Villalta M, Estévez A, Bransden MP, et al. (2008) Effects of dietary eicosapentaenoic acid on growth, survival, pigmentation and fatty acid composition in Senegal sole (Solea senegalensis) larvae during the Artemia feeding period. Aquacult Nutr 14, $232-241$.

13. Elango R, Ball RO \& Pencharz PB (2008) Indicator amino acid oxidation: concept and application. J Nutr 138, 243-246.

14. Folch J, Lees M \& Sloane Stanley GH (1957) A simple method for the isolation and purification of total lipides from animal tissues. J Biol Chem 226, 497-509.

15. Fox C (1990) Studies on polyunsaturated fatty acid nutrition in larvae of marine fish - the herring, Clupea harengus L. PhD Thesis, University of Stirling.

16. Izquierdo MS, Watanabe T, Takeuchi T, et al. (1990) Optimum EFA levels in Artemia to meet the EFA requirements of red sea bream (Pagrus major). In Current Status of Fish Nutrition in Aquaculture, pp. 221-232 [M Takeda and $\mathrm{T}$ Watanabe, editors]. Tokyo: Tokyo University Fisheries.

17. Rønnestad I, Rojas-García CR, Tonheim SK, et al. (2001) In vivo studies of digestion and nutrient assimilation in marine fish larvae. Aquaculture 201, 161-175.

18. Morais S, Koven W, Rønnestad I, et al. (2005) Dietary protein: lipid ratio and lipid nature affects fatty acid absorption and metabolism in a teleost larva. Br J Nutr 93, 813-820.

19. Zar JH (1996) Biostatistical Analysis. Upper Saddle River, NJ: Prentice Hall International.

20. Morais S, Narciso L, Dores E, et al. (2004) Lipid enrichment for Senegalese sole (Solea senegalensis) larvae: effect on larval growth, survival and fatty acid profile. Aquacult Int 12, 281-298.

21. Johnsen RI, Grahl-Nielsen O \& Roem A (2000) Relative absorption of fatty acids by Atlantic salmon Salmo salar from different diets, as evaluated by multivariate statistics. Aquacult Nutr 6, 255-261.

22. Sire M-F \& Vernier J-M (1981) Étude ultrastructurale de la synthèse de chylomicrons au cours de l'absorption intestinale des lipides chez la Truite. Influence de la nature des acides gras ingérés. Biol Cellulaire 40, 47-62.

23. Pérez JA, Rodríguez C \& Henderson RJ (1999) The uptake and esterification of radiolabelled fatty acids by enterocytes isolated from rainbow trout (Oncorhynchus mykiss). Fish Physiol Biochem 20, 125-134.

24. Geurden I, Bergot P, Schwarz L, et al. (1998) Relationship between dietary phospholipid classes and neutral lipid absorption in newly-weaned turbot, Scophthalmus maximus. Fish Physiol Biochem 19, 217-228. 